\title{
SUPPLEMENATRY INFORMATION
}

\author{
for
}

\section{Mitigating the Trade-off between Triplet Harvesting and Roll-off by Opening a Dexter-Type Channel in OLEDs}

Hyung Suk Kim ${ }^{\mathrm{a}}$, Sang Hoon Lee ${ }^{\mathrm{b}}$, Ja Yeon Lee ${ }^{\mathrm{b}}$, Seunghyup Yoo ${ }^{\text {a,* }}$, and Min Chul Suh ${ }^{\text {b,* }}$

${ }^{a}$ School of Electrical Engineering, Korea Advanced Institute of Science and Technology (KAIST), Daejeon 34141, Republic of Korea.

${ }^{b}$ Department of Information Display, Kyung Hee University, Seoul 02447, Republic of Korea.

*Corresponding Authors

E-mail: syoo.ee@kaist.edu

E-mail: $\underline{\text { mcsuh@khu.ac.kr }}$ 


\section{The Exciton Lifetime Fitting}

The transient intensities (I, counts) are fitted with the sum of exponential decay (i.e. mono- or bi-) of the following form ${ }^{1}$;

$$
I=\sum_{i=1}^{n} A_{\mathrm{n}} e^{-\frac{t}{\tau_{n}}}+A_{\mathrm{IRF}}
$$

Eq. (S1)

Where $A_{\mathrm{n}}$ is the relative contribution of the individual decays with lifetimes $\left(\tau_{\mathrm{n}}\right)$ and $A_{\mathrm{IRF}}$ is a constant offset corresponding to an instrument response function (IRF) in our system.

\section{The Definition of $\boldsymbol{k}_{\mathrm{RFET}}$ and $\boldsymbol{k}_{\mathrm{DET}}$ for an Optical Model (Steady-State Condition)}

The solution to Eq. (2) $-G$ and (3) is a bi-exponential decay ( $2^{\text {nd }}$ order linear differential equations) given by

$$
N_{\mathrm{S}}(t), N_{\mathrm{T}}(t)=A_{1} e^{-k_{\mathrm{PF}} t}+A_{2} e^{-k_{\mathrm{DF}} t}
$$

Eq. (S2)

where $k_{\mathrm{PF}}\left(k_{\mathrm{DF}}\right)$ is the measurable decay rates for prompt (delayed) collected by Tr-PL profile at each individual system, respectively. Using the Viète's theorem, we can simply define the rate constant for energy transfer factors, which can be expressed as

$$
k_{\mathrm{PF}}+k_{\mathrm{DF}}=k_{\mathrm{FRET}}+k_{\mathrm{r}}^{\mathrm{s}}+k_{\mathrm{nr}}^{\mathrm{s}}+k_{\mathrm{ISC}}+k_{\mathrm{DET}}+k_{\mathrm{nr}}^{\mathrm{T}}+k_{\mathrm{RISC}}
$$

Eq. (S3)

$$
k_{\mathrm{PF}} k_{\mathrm{DF}}=\left(k_{\mathrm{FRET}}+k_{\mathrm{r}}^{\mathrm{s}}+k_{\mathrm{nr}}^{\mathrm{s}}+k_{\mathrm{ISC}}\right)\left(k_{\mathrm{DET}}+k_{\mathrm{nr}}^{\mathrm{T}}+k_{\mathrm{RISC}}\right)-k_{\mathrm{RISC}} k_{\mathrm{ISC}}
$$

Eq. (S4)

Under the condition $\left(k_{\mathrm{PF}}>>k_{\mathrm{DF}}\right)$, Eq. (S3) gives:

$$
k_{\mathrm{FRET}} \cong k_{\mathrm{PF}}-k_{\mathrm{r}}^{\mathrm{s}}-k_{\mathrm{ISC}}
$$

Eq. (S5)

Further, we can describe exactly $k_{\text {FRET }}$ by introduction of FRET theory. ${ }^{2}$

$$
k_{\mathrm{FRET}}=1 / \tau_{\mathrm{D}}\left(\frac{R_{\mathrm{F}}}{R}\right)^{6}
$$

Eq. (S6)

Thus, combining Eq. (S5) with (S6), we can re-define $k_{\mathrm{PF} \text {,estimated }}$ as a function of $R$ reflecting on energy transfer at each system in accordance with the doping concentration. 


$$
k_{\mathrm{PF}, \text { estimated }}(R)=1 / \tau_{\mathrm{D}}\left(\frac{R_{\mathrm{F}}}{R}\right)^{6}+k_{\mathrm{r}}^{\mathrm{s}}+k_{\mathrm{ISC}}
$$

Eq. (S7)

Without any approximation, $k_{\mathrm{DET}}$ can be defined from Eq. (S4)

$$
k_{\mathrm{DET}}=\frac{k_{\mathrm{PF}} k_{\mathrm{DF}}-k_{\mathrm{RISC}}\left(k_{\mathrm{FRET}}+k_{\mathrm{r}}^{\mathrm{s}}+k_{\mathrm{nr}}^{\mathrm{s}}\right)}{k_{\mathrm{FRET}}+k_{\mathrm{r}}^{\mathrm{s}}+k_{\mathrm{nr}}^{\mathrm{s}}+k_{\mathrm{ISC}}}-k_{\mathrm{nr}}^{\mathrm{T}}
$$

Eq. (S8)

Meanwhile, $k_{\mathrm{DET}}$ also can be expressed by taking DET theory, ${ }^{3}$ which shows exponential dependent $R$.

$$
k_{\mathrm{DET}}=K J_{\mathrm{D}} e^{-\beta R}
$$

Eq. (S9)

where $K$ is a parameter associated with the specific orbital interactions, $J_{\mathrm{D}}$ is a spectral overlap integral normalized for the extinction coefficient of the ground state molecule, and $\beta$ is the attenuation coefficient. In general, we could get $k_{\mathrm{DET}}$ by fitting the values of $K J_{\mathrm{D}}$ and $\beta$ with respect to experimental results.

Instead, we here adopt the $\min \left[k_{\mathrm{DF}, \text { estimated }}\right]$ to classify the cases [Figure 2b] wherein the minimum value of $k_{\mathrm{DF}}$ to meet DET activation condition from Eq. (S8) (vide infra)

$$
\min \left[k_{\mathrm{DF}, \text { estimated }}\right] \geq \frac{k_{\mathrm{RISC}}\left(k_{\mathrm{FRET}}+k_{\mathrm{r}}^{\mathrm{s}}+k_{\mathrm{nr}}^{\mathrm{s}}\right)}{k_{\mathrm{PF}, \text { estimated }}}
$$

Eq. (S10)

$\min \left[k_{\mathrm{DF}, \text { estimated }}\right]$ should be not less than $k_{\mathrm{DF}, \mathrm{TADF}}$ for the validity of the present energy transfer system mathematically .

$$
\min \left[k_{\mathrm{DF}, \text { estimated }}\right] \geq k_{\mathrm{DF}, \mathrm{TADF}}
$$

Eq. (S11)

where the experimental value for $k_{\mathrm{DF}, \mathrm{TADF}}$ that we used to obtain photophysical rate constants related to TADF behavior. $k_{\mathrm{DF}}$,TADF (refer to Table S1) corresponds to the decay rates for the delayed profile in the system without the end dopant (i.e., $k_{\mathrm{DET}}=0 \mathrm{~s}^{-1}$ ). Thus, we could estimate $R_{\mathrm{D}}$ (DET starting point) where the DET process is initiated $\left[R_{\mathrm{D}} @ \min \left[k_{\mathrm{DF}, \text { estimated }}\right]=k_{\mathrm{DF}, \mathrm{TADF}}\right]$. 


\section{The Exciton Dynamics for an Electrical Model}

From the optical model, we can establish an electrical model by adding $J / 4 q W-G$ and $3 J / 4 q w$ in Eq. (2) and (3), which can be rearranged as follows (vide infra)

$$
\frac{d N_{\mathrm{S}}(t)}{d t}=-\left(k_{\mathrm{FRET}}+k_{\mathrm{r}}^{\mathrm{s}}+k_{\mathrm{nr}}^{\mathrm{s}}+k_{\mathrm{ISC}}\right) N_{\mathrm{S}}(t)+k_{\mathrm{RISC}} N_{\mathrm{T}}(t)+\frac{J}{4 q W}
$$

Eq. (S12)

$$
\frac{d N_{\mathrm{T}}(t)}{d t}=-\left(k_{\mathrm{DET}}+k_{\mathrm{nr}}^{\mathrm{T}}+k_{\mathrm{RISC}}\right) N_{\mathrm{T}}(t)+k_{\mathrm{ISC}} N_{\mathrm{S}}(t)+\frac{3 J}{4 q W}
$$

Eq. (S13)

\section{Steady-State Condition for an Electrical Model}

Under the steady state condition on the electrical model, i.e., $\frac{d N_{\mathrm{S}}(t)}{d t}=0$, and $\frac{d N_{\mathrm{T}}(t)}{d t}=0$, we can arrange the Eq. (S12) and (S13) as follows

$$
\lim _{t \rightarrow \infty} N_{\mathrm{S}}(t, J)=\frac{\frac{J}{4 q W}\left(k_{\mathrm{DET}}+k_{\mathrm{nr}}^{\mathrm{T}}+4 k_{\mathrm{RISC}}\right)}{\left(k_{\mathrm{FRET}}+k_{\mathrm{r}}^{\mathrm{S}}+k_{\mathrm{nr}}^{\mathrm{S}}+k_{\mathrm{ISC}}\right)\left(k_{\mathrm{DET}}+k_{\mathrm{nr}}^{\mathrm{T}}+k_{\mathrm{RISC}}\right)-k_{\mathrm{RISC}} k_{\mathrm{ISC}}}
$$

Eq. (S14)

$$
\lim _{t \rightarrow \infty} N_{\mathrm{T}}(t, J)=\frac{\frac{J}{4 q W}\left(4 k_{\mathrm{ISC}}+3 k_{\mathrm{r}}^{\mathrm{S}}+3 k_{\mathrm{nr}}^{\mathrm{S}}+3 k_{\mathrm{FRET}}\right)}{\left(k_{\mathrm{FRET}}+k_{\mathrm{r}}^{\mathrm{S}}+k_{\mathrm{nr}}^{\mathrm{S}}+k_{\mathrm{ISC}}\right)\left(k_{\mathrm{DET}}+k_{\mathrm{nr}}^{\mathrm{T}}+k_{\mathrm{RISC}}\right)-k_{\mathrm{RISC}} k_{\mathrm{ISC}}}
$$

Eq. (S15) 

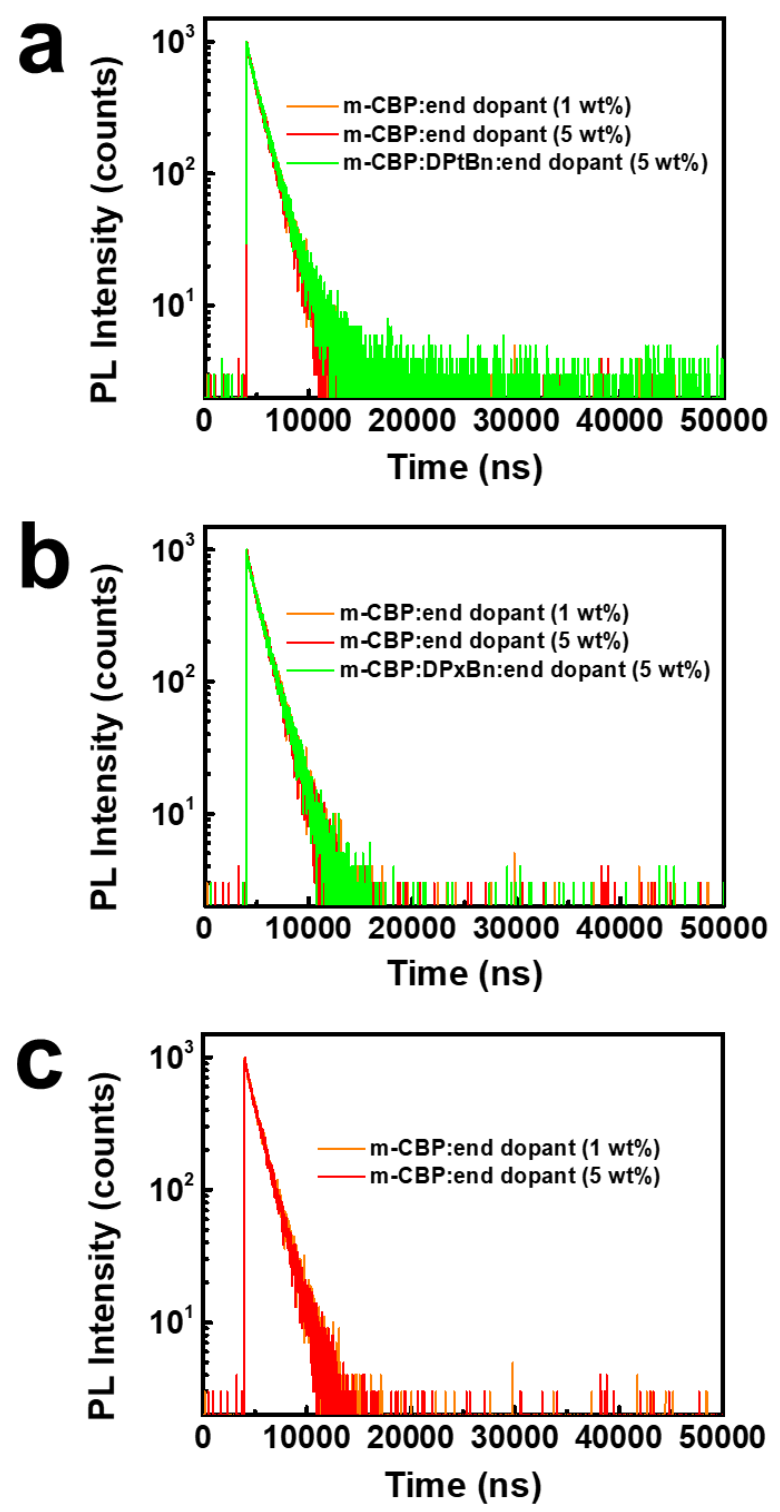

Figure. S1 Tr-PL data collected at $\lambda_{\text {peak }}$ [time-scale: $50 \mu \mathrm{s}$, excitation wavelength $\left(\lambda_{\text {ex }}\right): 340 \mathrm{~nm}$ ] for a various of EMLs [ $m$-CBP:DPtBn:Ir(phq $)_{2}(\mathrm{acac})$ and $m$-CBP:DPxBn:Ir(phq $)_{2}$ (acac)] corresponding to (a) and (b), respectively. (c) Tr-PL decay profiles for 1.0 and $5.0 \mathrm{wt} . \% \operatorname{Ir}(\mathrm{phq})_{2}$ (acac) doped $m$-CBP solid films were given to proceed the comparison between (a) and (b). 

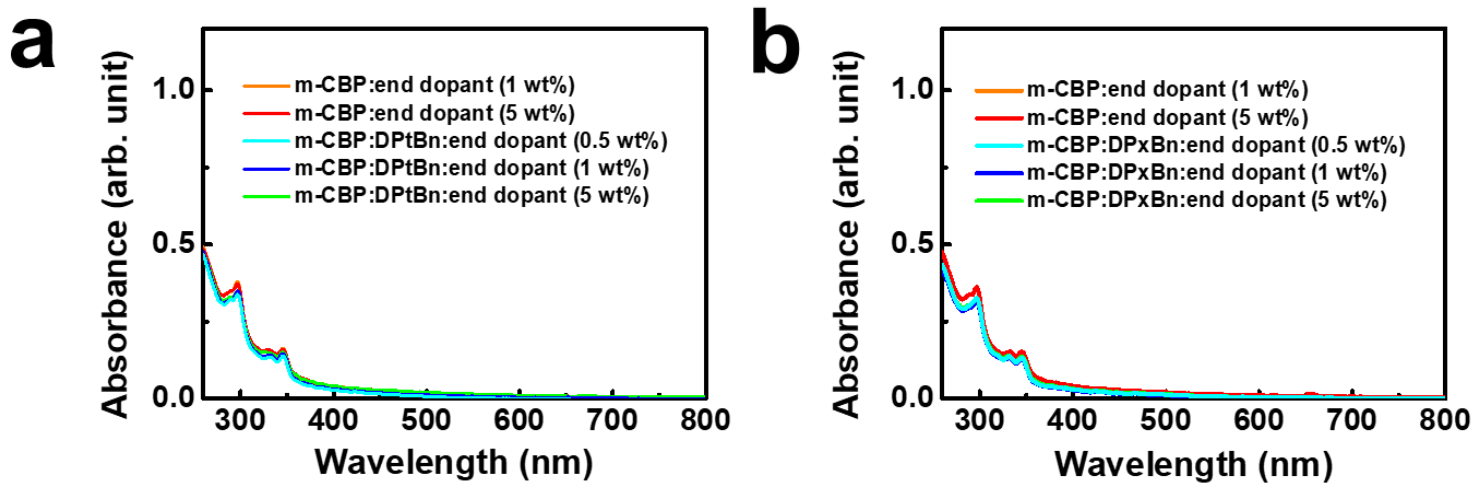

Figure. S2 Absorption spectra of each individual system based on (a) DPtBn and (b) DPxBn TADF materials (thickness: $30 \mathrm{~nm}$ ). 

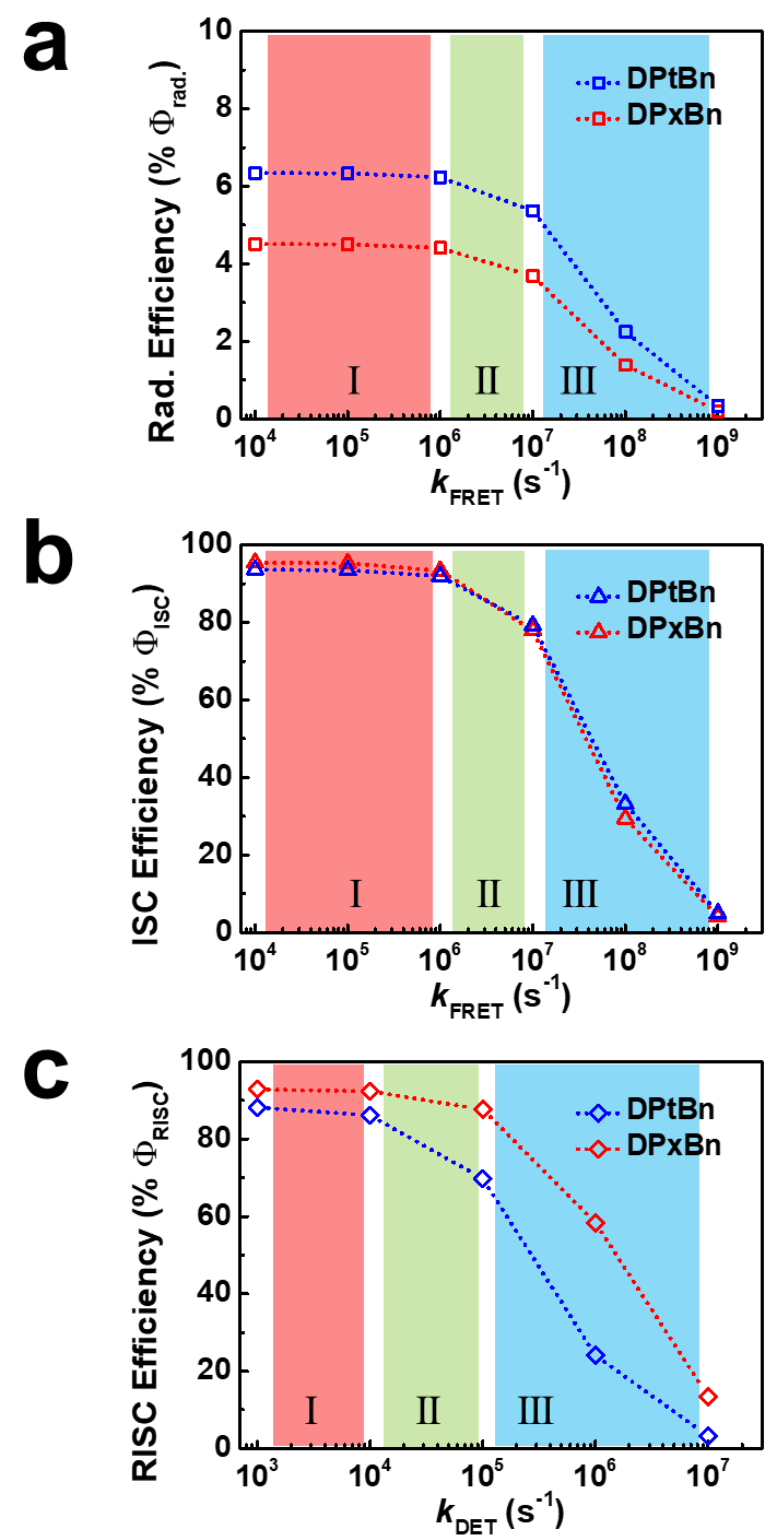

Figure. S3 (a) Radiative efficiency ( $\left.\Phi_{\text {Rad. }}\right)$ for DPtBn and DPxBn versus rate constant of FRET ( $k_{\mathrm{FRET}}$ : $10^{4}-10^{9} \mathrm{~s}^{-1}$ ). (b) ISC efficiency ( $\Phi_{\text {ISC }}$ ) for DPtBn and DPxBn depending on the value of $k_{\text {FRET. }}$ (c) RISC efficiency $\left(\Phi_{\text {RISC }}\right)$ for DPtBn and DPxBn upon the modulation of DET process $\left(k_{\mathrm{DET}}: 10^{3}-10^{7} \mathrm{~s}^{-1}\right)$. Area I, II, and III are divided on the basis of the change in efficiency, depending on the rate constant of FRET or DET. 

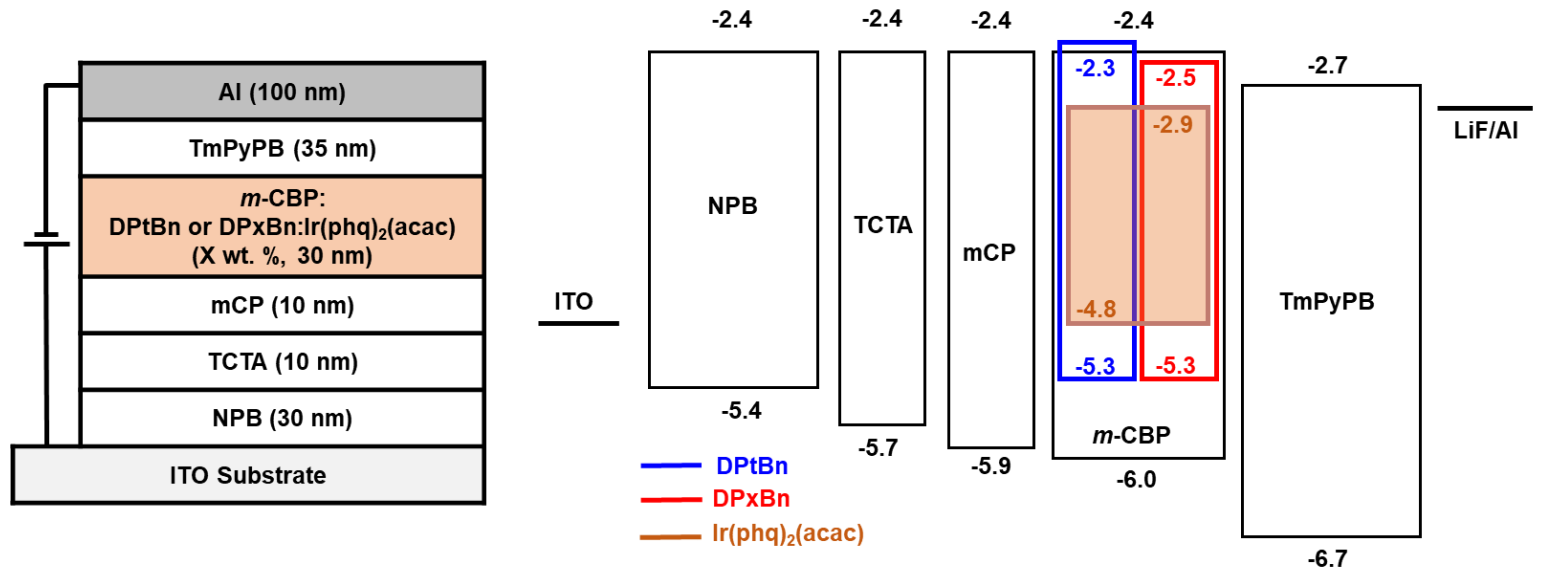

Figure. S4 Schematic of the device architecture (left) and energy diagram of the OLEDs utilized in this work (right).
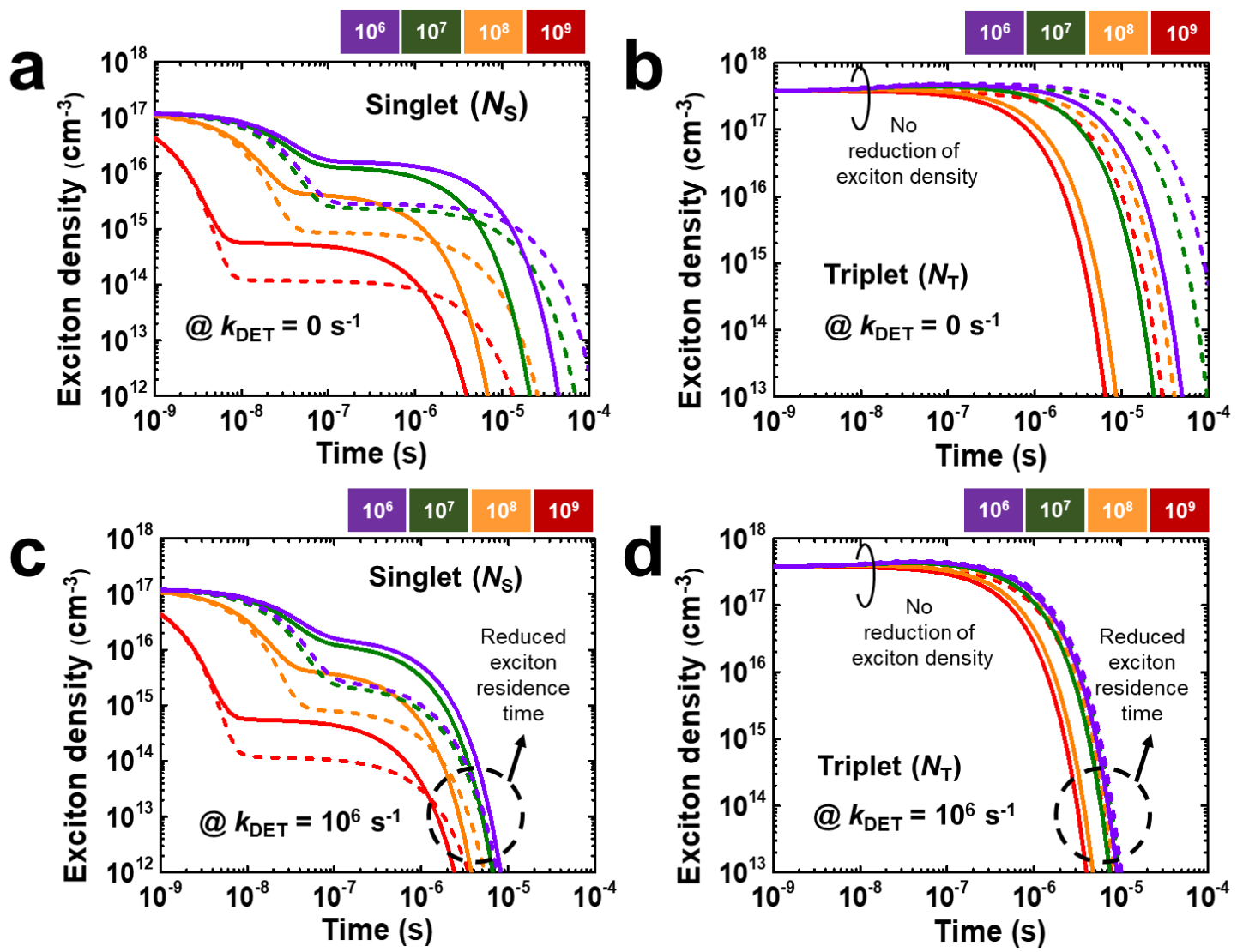

Figure. S5 Numerically calculated singlet density $\left[N_{\mathrm{S}}\right]$ (a) and triplet density $\left[N_{\mathrm{T}}\right]$ (b), in DPtBn (dotted line)/DPxBn (solid line): $m$-CBP co-deposited solid film, at the various rate constant of $k_{\text {FRET }}\left(10^{6}-10^{9}\right.$ $\mathrm{s}^{-1}$, violet, olive, orange, red are designated in order) after electrical excitation (the pulse width: $5 \mathrm{~ns}$ ). Calculated $N \mathrm{~s}(\mathrm{c})$ and $N_{\mathrm{T}}(\mathrm{d})$ as a function of time with various $k_{\mathrm{FRET}}$ (from $10^{6}$ to $10^{9} \mathrm{~s}^{-1}$ ) and an activation of DET $\left(k_{\mathrm{DET}}=10^{6} \mathrm{~s}^{-1}\right)$ using Eqs. $(\mathrm{S} 14)$ and $(\mathrm{S} 15)$. 

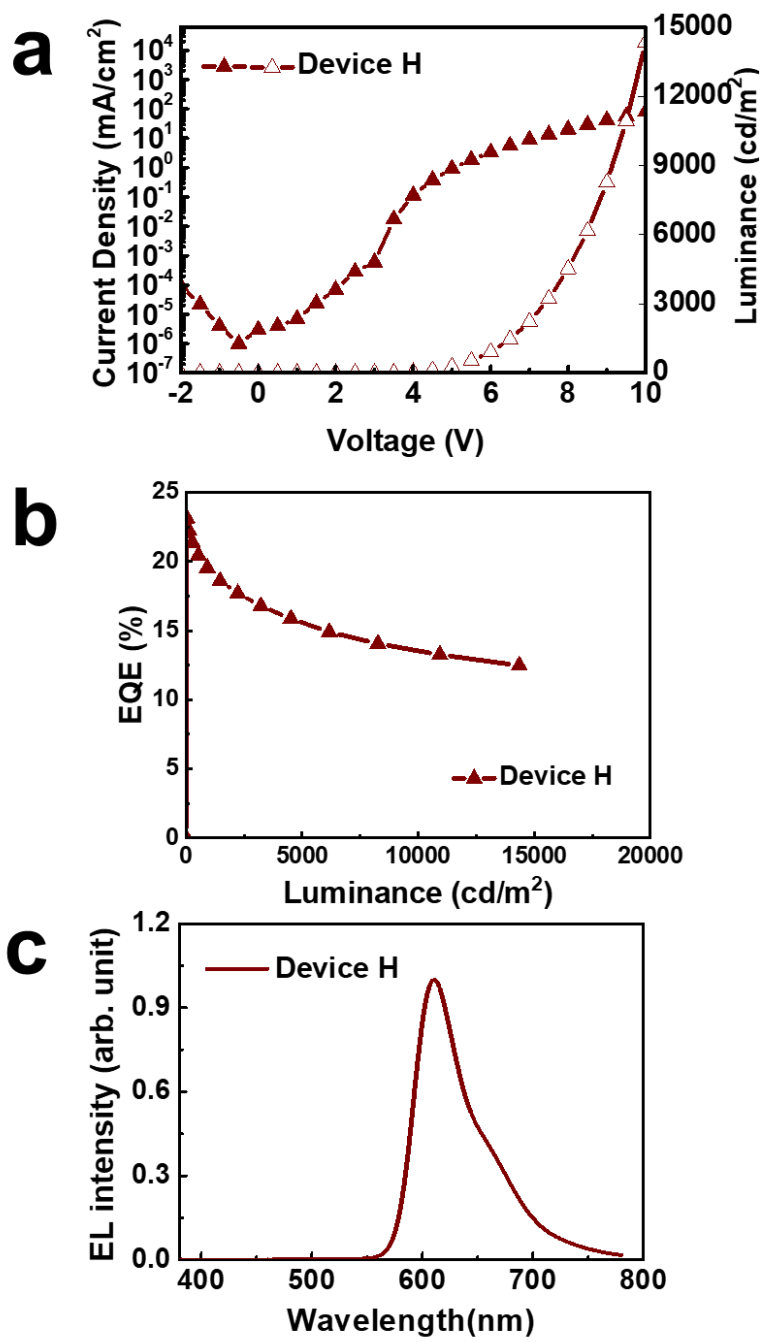

Figure. S6 (a) $J-V$ - $L$ (b) EQE-luminance curves, and (c) EL profile for device $\mathrm{H}$. 
Table S1. PL Characteristics and Rate Constants of Utilized TADF Molecules

\begin{tabular}{cccccccccc}
\hline $\begin{array}{c}\text { TADF } \\
\text { molecule }\end{array}$ & $\begin{array}{c}\tau_{\mathrm{PF}}{ }^{\mathrm{a}} \\
{[\mathrm{ns}]}\end{array}$ & $\begin{array}{c}\left.\tau_{\mathrm{DF}^{\mathrm{a}}}{ }^{\mathrm{C}} \mathrm{\mu \textrm {s }}\right]\end{array}$ & $\begin{array}{c}\Delta E_{\mathrm{ST}^{\mathrm{a}}} \\
{[\mathrm{eV}]}\end{array}$ & $\begin{array}{c}{ }^{1} \mathrm{CT}^{\mathrm{a}} \\
{[\mathrm{eV}]}\end{array}$ & $\begin{array}{c}{ }^{3} \mathrm{LE}_{\mathrm{D}}^{\mathrm{b}} \\
{[\mathrm{eV}]}\end{array}$ & $\begin{array}{c}k_{\mathrm{r}}^{\mathrm{S}} \\
{\left[10^{6} \mathrm{~s}^{-1}\right]}\end{array}$ & $\begin{array}{c}k_{\mathrm{nr}}{ }^{\mathrm{T}} \\
{\left[10^{5} \mathrm{~s}^{-1}\right]}\end{array}$ & $\begin{array}{c}k_{\mathrm{ISC}} \\
{\left[10^{7} \mathrm{~s}^{-1}\right]}\end{array}$ & $\begin{array}{c}k_{\mathrm{RISC}} \\
{\left[10^{6} \mathrm{~s}^{-1}\right]}\end{array}$ \\
\hline DPtBn & 18.2 & 15.6 & 0.14 & 2.44 & 2.30 & 3.49 & 0.43 & 5.15 & 0.33 \\
DPxBn & 22.5 & 5.3 & 0.05 & 2.45 & 2.34 & 2.00 & 1.20 & 4.24 & 1.57
\end{tabular}

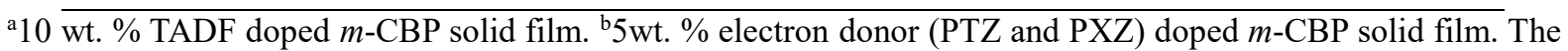
physical constants denoted here are referenced from the previous report. ${ }^{4}$

Table S2. The Efficiencies for DPtBn and DPxBn TADF molecules

\begin{tabular}{cccccc}
\hline $\begin{array}{c}\text { "TADF } \\
\text { molecule }\end{array}$ & $\begin{array}{c}\Phi_{\mathrm{ISC}} \\
{[\%]}\end{array}$ & $\begin{array}{c}\Phi_{\mathrm{RISC}} \\
{[\%]}\end{array}$ & $\begin{array}{c}\Phi_{\mathrm{PF}} \\
{[\%]}\end{array}$ & $\begin{array}{c}\Phi_{\mathrm{DF}} \\
{[\%]}\end{array}$ & $\begin{array}{c}\Phi_{\mathrm{PLQY}} \\
{[\%]}\end{array}$ \\
\hline DPtBn & 93.7 & 88.5 & 6 & 31 & 37 \\
DPxBn & 95.5 & 92.9 & 4 & 36 & 40
\end{tabular}

${ }^{*}$ The efficiency values for TADF molecules denoted here are referenced from the previous report. ${ }^{4}$

Table S3. Device Performance of Tested OLED (device H).

\begin{tabular}{|c|c|c|c|c|c|}
\hline \multirow[b]{2}{*}{ System } & \multirow{2}{*}{$\begin{array}{c}\text { End } \\
\text { dopant } \\
\text { (wt. \%) }\end{array}$} & \multirow{2}{*}{$\begin{array}{c}\mathrm{V}_{\mathrm{on}}^{\mathrm{a}} / \mathrm{V}_{\mathrm{op}}^{\mathrm{b}} \\
(\mathrm{V})\end{array}$} & \multicolumn{2}{|c|}{$\mathrm{CE} / / \mathrm{PE} / / \mathrm{EQE}(\mathrm{cd} / \mathrm{A} / / \mathrm{lm} / \mathrm{W} / / \%)$} & \multirow{2}{*}{$\operatorname{CIE}(x, y)^{\mathrm{t}}$} \\
\hline & & & Maximum & At $1,000 \mathrm{~cd} / \mathrm{m}^{2}$ & \\
\hline $\begin{array}{l}\text { SET in } \\
\text { DPxBn }^{c}\end{array}$ & 5.0 & $3.1 / 6.1$ & $31.7 / 27.0 / 23.4$ & 27.2/14.2/19.5 & $(0.65,0.35)$ \\
\hline
\end{tabular}

${ }^{\mathrm{a}}$ Measured at $1 \mathrm{~cd} / \mathrm{m}^{2} \mathrm{~b}$ Measured at $1000 \mathrm{~cd} / \mathrm{m}^{2} \mathrm{Cr}(\mathrm{mphmq})_{2}(\mathrm{tmd})$ was utilized for this system.

\section{Reference}

[1] Redondo, C. S.; Kleine, P.; Roszeitis, K.; Achenbach, T.; Kroll, M.; Thomschke, M.; Reineke, S. Interplay of Fluorescence and Phosphorescence in Organic Biluminescent Emitters J.Phys. Chem. C 2017, 121, 14946-14953.

[2] Förster, T. $10^{\text {th }}$ Spiers Memorial Lecture. Transfer Mechanisms of Electronic Excitation Discuss. Faraday Soc. 1959, 27, 7-17.

[3] Dexter, D. L. Theory of Sensitized Luminescence in Solids J. Chem. Phys. 1953, 21, 836-850.

[4] Kim, H. S.; Park, H.; Park, S. -R.; Lee, S. H.; Ahn, Y.; Lee, Y. S.; Suh, M. C. Photophysical Properties of Thermally Activated Delayed Fluorescent Materials upon Distortion of Central Axis of Donor Moiety J. Phys.

Chem. C 2018, 122, 28576-28587. 